\title{
Addendum
}

\section{On the Kuznetsov-Bruggeman formula for a Hilbert modular surface having one cusp}

\section{Joyner}

Department of Mathematics, U.S. Naval Academy, Annapolis, MD 21402, USA

Math. Z. 203, 59104 (1990)

There is a gap in the proof of

$$
\sum_{\substack{c \\ 0<c<T_{1} \\ 0<c<T_{2}}} \frac{S\left(v, v^{\prime},(c)\right)}{N(c)} \ll_{v, v^{\prime}, \varepsilon}\left(T_{1} T_{2}\right)^{1 / 6+\varepsilon}
$$

(Lemma 4.26) in the above-mentioned article. The proof of this estimate implicitly assumed that there are no exceptional eigenvalues for $S L_{2}\left(O_{K}\right) \backslash H^{2}$. The exceptional eigenvalues for $S L_{2}\left(\mathcal{O}_{K}\right) \backslash H^{2}$, if any, create additional terms in this estimate. The correct result is

Lemma. Assume the notation of the above-cited paper. We have

$$
\begin{aligned}
& \sum_{\substack{c \\
0<c<T_{1} \\
0<\varepsilon<T_{2}}} \frac{S\left(v, v^{\prime},(c)\right)}{N(c)} \ll_{D, v, v^{\prime}, \varepsilon}\left(T_{1} T_{2}\right)^{1 / 6+\varepsilon} \\
& +\sum_{\substack{\left.\left.j \\
0<j^{\prime}\right)<\frac{1}{2} \\
0<\lambda j^{\prime}\right)<\frac{1}{4}}}\left[\left(T_{1} T_{2}\right)^{2\left|r_{1 j}\right|+\varepsilon}+\left(T_{1} T_{2}\right)^{2\left|r_{2 j}\right|+\varepsilon}\right] .
\end{aligned}
$$

Proof. The proof is exactly the same except that the estimate (4.30), which implicitly assumes that there are no exceptional eigenvalues, must be replaced by

$$
\begin{aligned}
& \sum_{\substack{r_{1 j} \leq M_{1} \\
r_{2 j} \leqq M_{2}}}\left|\frac{\rho_{j}(v) \overline{\rho_{j}\left(v^{\prime}\right)}}{\cosh \left(\pi r_{1 j}\right) \cosh \left(\pi r_{2 j}\right)} h\left(r_{1 j}, r_{2 j}\right)\right| \\
& \ll\left(M_{1} M_{2}\right)^{1 / 2}+\sum_{\substack{\left.j \\
0<\lambda(1)<\frac{1}{4} \\
0<\lambda, 11\right)<\frac{1}{4}}}\left[\left(T_{1} T_{2}\right)^{2\left|r_{1 j}\right|+\varepsilon}+\left(T_{1} T_{2}\right)^{2\left|r_{2 j}\right|+\varepsilon}\right] .
\end{aligned}
$$

This follows from the estimates of bump functions in the article of Deshoullier and Iwaniec, Invent. Math. 70, pp. 270-273 (1982). 